August 3, 2009

OU-HET 631/2009

KIAS-P09040

\title{
Stable Higgs Bosons as Cold Dark Matter
}

\author{
Yutaka Hosotani, ${ }^{*}$ Pyungwon $\mathrm{Ko}^{\dagger}$ and Minoru Tanaka* \\ *Department of Physics, Osaka University, Toyonaka, Osaka 560-0043, Japan \\ ${ }^{\dagger}$ School of Physics, KIAS, Seoul 130-722, Korea
}

\begin{abstract}
In a class of the gauge-Higgs unification models the 4D neutral Higgs boson, which is a part of the extra-dimensional component of the gauge fields, becomes absolutely stable as a consequence of the gauge invariance and dynamically generated new parity, serving as a promising candidate for cold dark matter (CDM). We show that the observed relic abundance of cold dark matter is obtained in the $S O(5) \times U(1)$ model in the warped space with the Higgs mass around $70 \mathrm{GeV}$. The Higgs-nucleon scattering cross section is found to be close to the current CDMS II and XENON10 bounds in the direct detection of dark matter.
\end{abstract}


What constitutes dark matter in the universe? [1]-44 How does the Higgs boson interact with other particles? [5] These are two of the most important issues in current physics. We would like to point out that these two issues are related to each other, and indeed that they reflect two sides of the fact that Higgs bosons can be stable and become dark matter.

In the standard model (SM) of electroweak interactions, the electroweak (EW) symmetry is spontaneously broken by the Higgs field. However it is not clear at all if the Higgs boson appears as envisaged in the standard model. It is often argued that the naturalness and stability against radiative corrections to the mass of the Higgs field indicate the existence of supersymmetry underlying the nature. However, there are other scenarios with the naturalness. The gauge-Higgs unification scenario is one of them, in which the 4D Higgs field, $H(x)$, is a part of the extra-dimensional component of the gauge fields. It is identified as the fluctuation mode of the Aharonov-Bohm phase (Wilson line phase), $\theta_{H}$, in the extra-dimension. [6]-8]

It has been recently found in one of the gauge-Higgs unification models in the RandallSundrum warped space[9] that the Higgs mass $m_{H}$ comes out around $50 \mathrm{GeV}$ and the Higgs couplings with $W$ and $Z$ bosons, $W W H$ and $Z Z H$, and Yukawa couplings with fermions, $H \bar{\psi} \psi$, vanish at the value of $\theta_{H}= \pm \frac{1}{2} \pi$ where the effective potential for $\theta_{H}$ is minimized. [10, 11] If this is the case, the Higgs boson would become stable.

This prompts urgent questions. Does the Higgs couplings to the SM fermions and weak gauge bosons vanish to all order in perturbation theory? Is the value $\theta_{H}= \pm \frac{1}{2} \pi$ naturally achieved? Can the Higgs boson be absolutely stable? What physical consequences follow from stable Higgs bosons?

We show in this paper that the $W W H, Z Z H$, and $H \bar{\psi} \psi$ couplings exactly vanish and the Higgs boson becomes stable, provided that certain conditions for the matter content are satisfied in the $S O(5) \times U(1)$ gauge-Higgs unification in the Randall-Sundrum warped space.

This leads to an important consequence. Higgs bosons become viable candidates for cold dark matter (CDM) in the universe. They are copiously produced in the very early universe. As the annihilation rate of Higgs bosons falls below the expansion rate of the universe, the annihilation processes get frozen and the remnant Higgs bosons become dark matter.

The annihilation rates of Higgs bosons can be reliably estimated, once the masses of $W, Z$, and fermions are given. The precise value of the Higgs mass $m_{H}$, which arises as 
quantum effects of $\theta_{H}$, depends on the details of the model. In the model of ref. [10], $m_{H}$ is predicted to be $\sim 50 \mathrm{GeV}$. It is shown below that the observed cold dark matter density is obtained with $m_{H} \sim 70 \mathrm{GeV}$ in the $S O(5) \times U(1)$ model.

The distinctive feature in the gauge-Higgs unification is that the Higgs field $H(x)$ corresponds to the fluctuation of the Aharonov-Bohm (AB) phase, $\theta_{H}$, in the extra-dimensional space, which is given by the phase of the path-ordered Wilson line integral along a noncontractible loop $C$ in the extra-dimension with a coordinate $y, P \exp \left\{i g_{A} \int_{C} d y A_{y}(x, y)\right\}$. The effective Higgs interactions with the $W, Z$ bosons and fermions in the standard model at low energies are summarized in the $S O(5) \times U(1)$ model as [1]

$$
\begin{aligned}
& \mathcal{L}_{\mathrm{eff}}=-V_{\mathrm{eff}}\left(\hat{\theta}_{H}\right)-m_{W}^{2}\left(\hat{\theta}_{H}\right) W_{\mu}^{\dagger} W^{\mu}-\frac{1}{2} m_{Z}^{2}\left(\hat{\theta}_{H}\right) Z_{\mu} Z^{\mu}-\sum_{a, b} m_{a b}^{F}\left(\hat{\theta}_{H}\right) \bar{\psi}_{a} \psi_{b} \\
& \hat{\theta}_{H}(x)=\theta_{H}+\frac{H(x)}{f_{H}}, \quad f_{H}=\frac{2}{g_{A}} \sqrt{\frac{k}{z_{L}^{2}-1}} \sim \frac{2}{\sqrt{k L}} \frac{m_{\mathrm{KK}}}{\pi g} .
\end{aligned}
$$

Here $k$ and $L$ are two parameters specifying the Randall-Sundrum warped space. [9] The fundamental region in the fifth coordinate is $0 \leq y \leq L$. The AdS curvature in the bulk region is given by $\Lambda=-6 k^{2}$. The warp factor $z_{L}=e^{k L}$ and the Kaluza-Klein (KK) mass scale $m_{\mathrm{KK}}=\pi k z_{L}^{-1}$ are, typically, $10^{15}$ and $1.5 \mathrm{TeV}$, respectively. The $S O(5)$ gauge coupling $g_{A}$ is related to the $4 \mathrm{D} S U(2)_{L}$ weak coupling $g$ by $g=g_{A} / \sqrt{L}$.

The effective potential $V_{\text {eff }}\left(\hat{\theta}_{H}\right)$ is dynamically generated at the one-loop level, and is finite. 6] The mass functions $m_{W}\left(\hat{\theta}_{H}\right), m_{Z}\left(\hat{\theta}_{H}\right)$, and $m_{a b}^{F}\left(\hat{\theta}_{H}\right)$ appear at the tree level, which include contributions from heavy KK excited states in intermediate states. [11, 12, 13. The value of the $\mathrm{AB}$ phase $\theta_{H}$ is dynamically determined by the location of the global minimum of $V_{\text {eff }}\left(\theta_{H}\right)$. There the fermion fields are diagonalized in the mass basis such that $m_{a b}^{F}=m_{a}^{F} \delta_{a b}$.

In the $S O(5) \times U(1)$ model in the RS warped space [14, 12, 15] the orbifold boundary conditions

$$
\left(\begin{array}{c}
A_{\mu} \\
-A_{y}
\end{array}\right)\left(x, y_{j}-y\right)=P_{j}\left(\begin{array}{c}
A_{\mu} \\
A_{y}
\end{array}\right)\left(x, y_{j}+y\right) P_{j}^{-1}, \quad(j=0,1)
$$

are imposed for $S O(5)$ gauge fields, where $\left(y_{0}, y_{1}\right)=(0, L)$. Here $P_{j}$ 's are elements of $S O(5)$. With the parity matrices $P_{j}=P_{j}^{\text {vec }}=\operatorname{diag}(-1,-1,-1,-1,1)$ in the vectorial representation, $S O(5)$ is broken to $S O(4) \simeq S U(2)_{L} \times S U(2)_{R}$. The $4 \mathrm{D}$ Higgs fields $\phi_{a}(x)$ $(a=1, \cdots, 4)$ appear as zero modes in the $S O(5) / S O(4)$ part of $A_{y}(x, y)$. In components $A_{y}^{\hat{a}}(x, y)=\phi_{a}(x) h_{0}(y)+\cdots$ where $h_{0}(y)$ is the zero mode wave function given below. 
An $S O(4)$ vector $\phi_{a}$ forms an $S U(2)_{L}$ doublet corresponding to the Higgs doublet in the standard model. $\phi_{1}, \phi_{2}, \phi_{3}$ are absorbed by $W$ and $Z$, whereas $\phi_{4}$ corresponds to the physical neutral Higgs field.

Let us denote the generators of $S O(5) / S O(4)$ by $T^{\hat{a}}(a=1, \cdots, 4)$. In the vectorial representation $\left(T_{\mathrm{vec}}^{\hat{4}}\right)_{a b}=(i / \sqrt{2})\left(\delta_{a 5} \delta_{b 4}-\delta_{a 4} \delta_{b 5}\right)$, whereas in the spinorial representation $T_{\mathrm{sp}}^{\hat{4}}=(1 / 2 \sqrt{2}) I_{2} \otimes \tau_{1} \cdot\left[14\right.$. The difference in normalization $\operatorname{Tr} T_{\mathrm{vec}}^{\hat{a}} T_{\mathrm{vec}}^{\hat{b}}=2 \operatorname{Tr} T_{\mathrm{sp}}^{\hat{a}} T_{\mathrm{sp}}^{\hat{b}}=\delta_{a b}$ becomes important in the subsequent discussions.

The fifth dimension in the Randall-Sundrum warped space has topology of the orbifold $S^{1} / Z_{2}$. Its metric satisfies $g_{M N}(x, y+2 L)=g_{M N}(x, y)=g_{M N}(x,-y)$. Consequently the $\mathrm{AB}$ phase $\theta_{H}$ along the fifth dimension is given by $\exp \left\{\frac{i}{2} \theta_{H}\left(2 \sqrt{2} T^{\hat{4}}\right)\right\}=$ $\exp \left\{i g_{A} \int_{0}^{L} d y\left\langle A_{y}\right\rangle\right\}$. The relevant part of the gauge potential becomes

$$
A_{y}(x, y)=\hat{\theta}_{H}(x) \cdot \sqrt{\frac{4 k}{z_{L}^{2}-1}} h_{0}(y) \cdot T^{\hat{4}}+\cdots,
$$

where $h_{0}(y)=\left[2 k /\left(z_{L}^{2}-1\right)\right]^{1 / 2} e^{2 k y}(0 \leq y \leq L)$ and $\hat{\theta}_{H}(x)$ is defined in (11). $h_{0}(y)$ is normalized by $\int_{0}^{L} d y e^{-2 k y} h_{0}(y)^{2}=1$, and is given, outside the fundamental region, by $h_{0}(-y)=h_{0}(y)=h_{0}(y+2 L)$.

$\theta_{H}$ is a phase variable. All the functions of $\hat{\theta}_{H}$ in the effective interaction in (11) are periodic with a period $2 \pi$. This periodicity follows from the large gauge invariance. 8, 16. 17] Given the boundary conditions (2), there remains the residual gauge invariance $A_{M} \rightarrow A_{M}^{\prime}=\Omega A_{M} \Omega^{-1}-\left(i / g_{A}\right) \Omega \partial_{M} \Omega^{-1}$ which preserves the boundary conditions. In general, new gauge potentials satisfy new boundary conditions

$$
\left(\begin{array}{c}
A_{\mu}^{\prime} \\
-A_{y}^{\prime}
\end{array}\right)\left(x, y_{j}-y\right)=P_{j}^{\prime} A_{M}^{\prime}\left(x, y_{j}+y\right) P_{j}^{\prime-1}-\frac{i}{g_{A}} P_{j}^{\prime} \partial_{M} P_{j}^{\prime-1},
$$

where $P_{j}^{\prime}=\Omega\left(x, y_{j}-y\right) P_{j} \Omega\left(x, y_{j}+y\right)^{-1}$. The residual gauge invariance is defined with $\Omega(x, y)$ satisfying $P_{j}^{\prime}=P_{j}$.

Consider a large gauge transformation $\Omega^{\text {large }}(y)=\exp \left\{i \alpha \int_{0}^{y} d y \sqrt{4 k /\left(z_{L}^{2}-1\right)} h_{0}(y)\right.$. $\left.T^{\hat{4}}\right\}$ in the spinorial representation in which $P_{j}^{\mathrm{sp}}=I_{2} \otimes \tau_{3}$. It $\operatorname{shifts} \hat{\theta}_{H}(x)$ to $\hat{\theta}_{H}^{\prime}(x)=$ $\hat{\theta}_{H}(x)-\alpha$. It is straightforward to see that the condition $P_{j}^{\prime \text { sp }}=P_{j}^{\text {sp }}$ is satisfied if $\alpha=2 \pi n$ ( $n$ : an integer). In other words all physical quantities must be periodic in $\theta_{H}$ with a period $2 \pi$.

Fermions in the vector representation of $S O(5)$, for instance, obey $\Psi\left(x, y_{j}-y\right)=$ $P_{j}^{\text {vec }} \gamma^{5} \Psi\left(x, y_{j}+y\right)$. Under a gauge transformation $\Omega^{\text {large }}(y)$ with $\alpha=\pi$, one finds that 
$\left(P_{0}^{\prime \text { vec }}, P_{1}^{\text {/vec }}\right)=\left(P_{0}^{\text {vec }}, P_{1}^{\text {vec }}\right)$ whereas $\left(P_{0}^{\prime \text { sp }}, P_{1}^{\prime \text { sp }}\right)=\left(P_{0}^{\text {sp }},-P_{1}^{\text {sp }}\right)$. Hence, if there are no fermions in the spinor representation of $S O(5)$, there appears enhanced symmetry. In this case all physical quantities become periodic in $\theta_{H}$ with a reduced period $\pi$. In the model of ref. [10] bulk fermions appear only in the vector representation, thereby this condition being satisfied. Brane fermions located at $y=0$ are not affected by the transformation as $\Omega^{\operatorname{large}}(0)=1$.

There is mirror reflection symmetry in the extra dimension. The action in the $\mathrm{RS}$ warped spacetime is invariant under $\left(x^{\mu}, y\right) \rightarrow\left(x^{\prime \mu}, y^{\prime}\right)=\left(x^{\mu},-y\right), A_{M}(x, y) \rightarrow$ $A_{M}^{\prime}\left(x^{\prime}, y^{\prime}\right)=\left(A_{\mu},-A_{y}\right)(x, y)$, and $\Psi(x, y) \rightarrow \Psi^{\prime}\left(x^{\prime}, y^{\prime}\right)= \pm \gamma^{5} \Psi(x, y)$. The orbifold boundary conditions are preserved under this transformation. Since $h_{0}(-y)=h_{0}(y)$, this implies that the theory at low energies is invariant under $\hat{\theta}_{H}(x) \rightarrow \hat{\theta}_{H}^{\prime}\left(x^{\prime}\right)=-\hat{\theta}_{H}(x)$.

In a class of the $S O(5) \times U(1)$ gauge-Higgs unification models in the warped space which contains fermions only in tensorial representations, but not in spinorial representations, of $S O(5)$, one can draw an important conclusion about the couplings of Higgs bosons. It follows from the enhanced gauge symmetry and mirror reflection symmetry that

$$
\begin{aligned}
& V_{\mathrm{eff}}\left(\hat{\theta}_{H}+\pi\right)=V_{\mathrm{eff}}\left(\hat{\theta}_{H}\right)=V_{\mathrm{eff}}\left(-\hat{\theta}_{H}\right), \\
& m_{W, Z}^{2}\left(\hat{\theta}_{H}+\pi\right)=m_{W, Z}^{2}\left(\hat{\theta}_{H}\right)=m_{W, Z}^{2}\left(-\hat{\theta}_{H}\right), \\
& m_{a b}^{F}\left(\hat{\theta}_{H}+\pi\right)=-m_{a b}^{F}\left(\hat{\theta}_{H}\right)=m_{a b}^{F}\left(-\hat{\theta}_{H}\right) .
\end{aligned}
$$

$m_{W}(0)=m_{Z}(0)=0$ as the EW symmetry is recovered at $\theta_{H}=0$. The set of fermion masses $\left\{-m_{a b}^{F}\right\}$ gives the same physics as the set $\left\{m_{a b}^{F}\right\}$ does. The relative signs in the equalities for $m_{a b}^{F}$ have been fixed by explicit evaluation at the tree level.[1]

It has been shown in ref. [10] that $V_{\text {eff }}\left(\theta_{H}\right)$ is minimized precisely at $\theta_{H}= \pm \frac{1}{2} \pi$ due to the contribution from the top quark in the RS spacetime. The relations in (5), then, imply that all of the functions $V_{\text {eff }}\left(\hat{\theta}_{H}\right), m_{W, Z}^{2}\left(\hat{\theta}_{H}\right)$, and $m_{a b}^{F}\left(\hat{\theta}_{H}\right)$ satisfy a relation $F\left(\frac{1}{2} \pi+f_{H}^{-1} H\right)=$ $F\left(\frac{1}{2} \pi-f_{H}^{-1} H\right)$. They are even functions of $H$ when expanded around $\theta_{H}= \pm \frac{1}{2} \pi$.

It follows that all odd-power Higgs couplings $H^{2 \ell+1}, H^{2 \ell+1} W_{\mu}^{\dagger} W^{\mu}, H^{2 \ell+1} Z_{\mu} Z^{\mu}$, and $H^{2 \ell+1} \bar{\psi}_{a} \psi_{b}$, vanish. In particular, the vanishing $W W H$ and $Z Z H$ couplings signal significant deviation from the standard model. Even if $m_{H}<m_{W}$, the LEP2 bound for the Higgs mass is evaded. Further, Yukawa couplings for $H \bar{\psi}_{a} \psi_{b}$ operators vanish identically, too.

The derivative couplings such as $\partial_{\mu} \hat{\theta}_{H} \bar{\psi} \gamma^{\mu} \gamma^{5} \psi$ and $\partial_{\mu} \hat{\theta}_{H} Z_{\nu}\left(\partial^{\mu} Z^{\nu}-\partial^{\nu} Z^{\mu}\right)$ are all forbidden by the mirror reflection symmetry. We observe that the effective interactions at low 
energies are invariant under $H(x) \rightarrow-H(x)$ with all other fields kept intact at $\theta_{H}= \pm \frac{1}{2} \pi$. We call it the $H$-parity. Among low energy fields only the Higgs field is $H$-parity odd. The Higgs boson becomes stable, protected by the $H$-parity conservation. We stress that the $H$-parity has emerged dynamically, unlike in the models of refs. [18, 19] where an additional Higgs doublet with odd parity is introduced by hand.

The mass functions are evaluated in the RS space. It is found in refs. [10, 11, 12] that, to a good approximation,

$$
\begin{aligned}
& m_{W}\left(\hat{\theta}_{H}\right) \sim \cos \theta_{W} m_{Z}\left(\hat{\theta}_{H}\right) \sim \frac{1}{2} g f_{H} \sin \hat{\theta}_{H}, \\
& m_{a}^{F}\left(\hat{\theta}_{H}\right) \sim \lambda_{a} \sin \hat{\theta}_{H},
\end{aligned}
$$

where $\theta_{W}$ is the weak mixing angle and the fermion mass matrix has been approximated by a diagonal one $m_{a b}^{F}=m_{a}^{F} \delta_{a b}$. If a fermion belongs to spinor representation of $S O(5)$, one would obtain $m_{a}^{F} \sim \lambda_{a} \sin \frac{1}{2} \hat{\theta}_{H}$. As $\theta_{H}=\frac{1}{2} \pi$, one finds that $m_{W} \sim \frac{1}{2} g f_{H}$ and $m_{a}^{F} \sim \lambda_{a}$. The value of $f_{H}$ is given by $f_{H} \sim 246 \mathrm{GeV}$. We note that this differs from the vev of the Higgs field $f_{H} \theta_{H}$.

Inserting (6) into (11), one finds the various Higgs couplings;

$$
\mathcal{L}_{\mathrm{eff}} \sim-\left\{m_{W}^{2} W_{\mu}^{\dagger} W^{\mu}+\frac{1}{2} m_{Z}^{2} Z_{\mu} Z^{\mu}\right\} \cos ^{2} \frac{H}{f_{H}}-\sum_{a} m_{a} \bar{\psi}_{a} \psi_{a} \cos \frac{H}{f_{H}} .
$$

The $W W H H$ coupling is given by $\frac{1}{4} g^{2} W_{\mu}^{\dagger} W^{\mu} H^{2}$, which is $(-1)$ times the coupling in the standard model 1 This coupling includes contributions coming from tree diagrams containing KK excited states $W_{n}$ of $W$ in the intermediate states with two vertices $W W_{n} H$. [13] The $\bar{\psi} \psi H^{2}$ coupling is given by $\left(m_{a} / 2 f_{H}^{2}\right) \bar{\psi}_{a} \psi_{a} H^{2}$. It is generated by two vertices $\psi \psi_{n} H$ where $\psi_{n}$ is the $n$-th KK excited state of $\psi$. One comment is in order. The approximate formula for the fermion mass function in (6) may need corrections, depending on the details of the model. The symmetry property leads, in general, to $m\left(\hat{\theta}_{H}\right)=\sum_{n=0}^{\infty} b_{2 n+1} \sin \left[(2 n+1) \hat{\theta}_{H}\right]$. Accordingly the $\bar{\psi} \psi H^{2}$ coupling constant may be altered.

Gauge-Higgs unification models under consideration are characterized with two parameters $f_{H}$ and $m_{H}$ at low energies. In a minimal model in the RS warped space, $f_{H}$ is fixed around $246 \mathrm{GeV}$ by $m_{W} \sim \frac{1}{2} g f_{H}$. The value of $m_{H}$, on the other hand, depends on the details of the matter content in the models. In the following numerical analysis, we fix $f_{H}=246 \mathrm{GeV}$, whereas $m_{H}$ is treated as a free parameter.

\footnotetext{
${ }^{1}$ We use diag. $(-+++)$ as 4 D Minkowski metric.
} 
With all the Higgs couplings at hand, one can estimate the annihilation rates of Higgs bosons in the early universe to determine its relic abundance as the cold dark matter. A rough estimate may be made with the following formula: $\Omega_{H} h^{2} \simeq 3 \times 10^{-27} \mathrm{~cm}^{3} \mathrm{~s}^{-1} /\langle\sigma v\rangle$, where $\Omega_{H}$ is the present mass density of the Higgs boson normalized by the critical density, $h$ denotes the Hubble constant in units of $100 \mathrm{~km} \mathrm{~s}^{-1} \mathrm{Mpc}^{-1}$, and $\langle\sigma v\rangle$ is the thermal average of the total annihilation cross section of the Higgs bosons multiplied by the relative velocity. The present mass density of cold dark matter is determined by WMAP collaboration as $\Omega_{\mathrm{CDM}} h^{2}=0.1131 \pm 0.0034$. [2]

Suppose that the Higgs mass is sufficiently smaller than $m_{W}$. The dominant annihilation process is $H H \rightarrow b \bar{b}$, and the abundance based on the above formula turns out much larger than the WMAP value. If the Higgs boson is heavier than $W, H H \rightarrow W^{+} W^{-}$ dominates, and the relic abundance turns out much smaller than the WMAP value. Thus, we expect that a Higgs boson slightly lighter than the $\mathrm{W}$ boson explains the cold dark matter abundance observed by WMAP.

To determine a favored Higgs mass precisely, we have employed a more elaborated formula to evaluate the relic abundance [3, 20, 4]. The annihilation rate per unit particle number density $\sigma v$ is expanded in a non-relativistic manner as $\sigma v=a+b v^{2}+O\left(v^{4}\right)$, and the relic abundance is given by $\Omega_{H} h^{2} \simeq 2.82 \times 10^{8} Y_{\infty}\left(m_{H} / \mathrm{GeV}\right)$ where $Y_{\infty}^{-1}=0.264 g_{*}^{1 / 2} m_{\mathrm{pl}} m_{H}\{a+$ $\left.3(b-a / 4) / x_{f}\right\} / x_{f}$. The freeze-out parameter $x_{f}=m_{H} / T_{f}$, where $T_{f}$ is the freeze-out temperature, is determined by $x_{f}=\ln \left[0.0382 m_{\mathrm{pl}}\left(a+6 b / x_{f}\right) c(2+c) m_{H} /\left(g_{*} x_{f}\right)^{1 / 2}\right]$ with $c \simeq 0.5$.

We take the following annihilation modes into account: $b \bar{b}, V^{(*)} V^{(*)}, \tau \bar{\tau}$ and $c \bar{c}$. Here $V$ denotes $W$ or $Z$, and $V^{*}$ means a virtual gauge boson that eventually goes into a pair of a fermion and an anti-fermion. Since we are interested in the threshold region of the $W W$ final state, the inclusion of 3-body and 4-body final states via virtual gauge boson(s) is mandatory.

The cross section of the annihilation process into a final state $X, \sigma(H H \rightarrow X)$, is obtained from the decay rate into $X, \Gamma(H \rightarrow X)$, in the standard model [5], by replacing the relevant vertices in the standard model by those in the present model, with an appropriate change in kinematical factors. For example, the cross section for $H H \rightarrow f \bar{f}$ is given by

$$
\sigma_{f \bar{f}}=\frac{N_{c}}{8 \pi \bar{\beta}_{i}} \frac{m_{f}^{2}}{f_{H}^{4}}\left(1-\frac{4 m_{f}^{2}}{s}\right)^{3 / 2}
$$




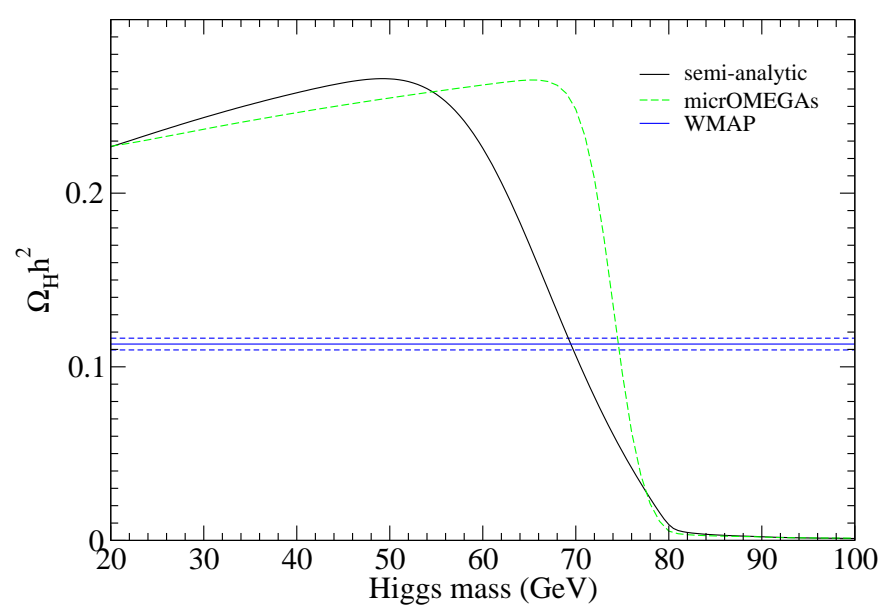

Figure 1: Thermal relic density of Higgs boson DM with $f_{H}=246 \mathrm{GeV}$. The solid (black) curve is obtained by the semi-analytic formulae, whereas the dashed (green) curve by the micrOMEGAs. The horizontal band is the WMAP data $\Omega_{\mathrm{CDM}} h^{2}=0.1131 \pm 0.0034$.

where $\sqrt{s}$ is the center-of-mass energy, $\bar{\beta}_{i}=\sqrt{1-4 m_{H}^{2} / s}$, and $N_{c}$ is the number of colors. The annihilation rate for $H H \rightarrow V^{(*)} V^{(*)}$ is given by

$$
\sigma_{V^{(*)} V^{(*)}}=\frac{1}{\pi^{2}} \int_{0}^{s} d q_{1}^{2} D_{V}\left(q_{1}^{2}\right) \int_{0}^{\left(\sqrt{s}-\sqrt{q_{1}^{2}}\right)^{2}} d q_{2}^{2} D_{V}\left(q_{2}^{2}\right) \sigma_{0}
$$

where $D_{V}\left(q^{2}\right)=m_{V} \Gamma_{V} /\left\{\left(q^{2}-m_{V}^{2}\right)^{2}+m_{V}^{2} \Gamma_{V}^{2}\right\}$, and $\sigma_{0}$ is the annihilation cross section into a pair of intermediate virtual vector bosons, given by $\sigma_{0}=\left(G_{F}^{2} s / 16 \pi \bar{\beta}_{i}\right) \delta_{V} \lambda^{1 / 2}\left[\lambda+12 q_{1}^{2} q_{2}^{2} / s^{2}\right]$ with $\delta_{W(Z)}=2(1)$, and $\lambda\left(q_{1}^{2}, q_{2}^{2} ; s\right)=s^{-2}\left\{\left(s-q_{1}^{2}-q_{2}^{2}\right)^{2}-4 q_{1}^{2} q_{2}^{2}\right\}$.

Fig. 1 shows our numerical results for the relic abundance of the Higgs CDM. The solid (black) curve is obtained by the semi-analytic formulae with all the aforementioned channels included in the total annihilation rate. The horizontal (blue) lines near 0.1 indicate the allowed range by WMAP. The favored Higgs mass is found around $70 \mathrm{GeV}$ in a rather narrow range. The freeze-out parameter is $x_{f} \sim 21$ in the favored region, which corresponds to $T_{f} \sim 3 \mathrm{GeV}$.

At $m_{H}=70 \mathrm{GeV}$, the annhilation rates of the relevant modes in the non-relativistic limit are $\sigma v=(7.3,11,1.5) \times 10^{-27} \mathrm{~cm}^{3} / \mathrm{s}$ for $b \bar{b}, W^{(*)} W^{(*)}$, and $Z^{(*)} Z^{(*)}$ respectively. The $W^{(*)} W^{(*)}$ mode is larger than the $b \bar{b}$ mode even below the $W W$ threshold, which confirms the importance of the $W^{(*)} W^{(*)}$ mode. All the remaining modes including the $g g$ mode have smaller rates.

Importance of the 3-body and 4-body final states through $H H \rightarrow V^{(*)} V^{(*)}$ below the threshold of $V V$ pair can be seen by comparing the solid (black) curve with the dashed 
(green) curve, which was obtained using the micrOMEGAs 2.2 [21] with two-body final states only. The $W W$ channel opens even below the threshold due to the thermal energy. The micrOMEGAs including only 2-body final states gives $m_{H} \simeq 75 \mathrm{GeV}$ as a favored Higgs mass. The relative contributions of the $b \bar{b}$ and $W W$ modes are $34 \%$ and $61 \%$, respectively. The result obtained from the semi-analytic formulae agrees with the result from the micrOMEGAs with only 2-body final states well below the $W W$ threshold. However, near the $W W$ threshold, the relic density from micrOMEGAs with 2-body final states in the $H H$ annihilation yields substantially larger $\Omega_{H} h^{2}$ than the semi-analytic treatment, although it includes thermally allowed 2-body final state $H H \rightarrow W W$. Near the $W W$ threshold, it is important to include the virtual $W$ effect correctly in order to get an accurate behavior of the relic density across the threshold.

If Higgs bosons constitute the cold dark matter of the universe, they can be detected by observing Higgs-nucleon elastic scattering process, $H N \rightarrow H N$. The relevant part of the effective interaction (7) is $\mathcal{L}_{\text {eff }}=\left(H^{2} / 2 f_{H}^{2}\right) \sum_{f} m_{f} \bar{f} f$.

To evaluate the direct detection rate one needs to incorporate QCD corrections [22]. After integrating out the heavy quarks, we obtain the effective Lagrangian at a hadronic scale:

$$
\mathcal{L}_{\text {eff }} \simeq \frac{H^{2}}{2 f_{H}^{2}}\left[\sum_{q=u, d, s} m_{q} \bar{q} q-\frac{\alpha_{s}}{4 \pi} G_{\mu \nu}^{a} G^{a \mu \nu}\right]
$$

where $c, b$ and $t$ quarks are integrated out, and $G_{\mu \nu}^{a}$ denotes the gluon field strength.

This effective Lagrangian leads to the following effective Higgs-nucleon coupling:

$$
\mathcal{L}_{H N} \simeq \frac{2+7 f_{N}}{9} \frac{m_{N}}{2 f_{H}^{2}} H^{2} \bar{N} N,
$$

where $f_{N}=\sum_{q=u, d, s} f_{q}^{N}$ and $\left\langle N\left|m_{q} \bar{q} q\right| N\right\rangle=m_{N} f_{q}^{N}$. The relation $\left\langle N\left|\left(\alpha_{s} / 8 \pi\right) G G\right| N\right\rangle=$ $-\left(m_{N} / 9\right)\left(1-f_{N}\right)$ has been used. With this coupling, the spin-independent (SI) Higgsnucleon scattering cross section is evaluated to be

$$
\sigma_{\mathrm{SI}} \simeq \frac{1}{4 \pi}\left(\frac{2+7 f_{N}}{9}\right)^{2} \frac{m_{N}^{4}}{f_{H}^{4}\left(m_{H}+m_{N}\right)^{2}},
$$

in the non-relativistic limit. There is a considerable uncertainty in the value of $f_{N}$ stemming from that in the $\sigma_{\pi N}$ term. The value $f_{N}$ quoted in ref.'s [21] and [23] are $f_{N} \sim(0.31 \sim 0.41)$ and $f_{N} \sim(0.20 \sim 0.45)$, respectively. On the other hand, the recent lattice calculation gives a smaller value for $f_{s}^{N}$ and $f_{N} \sim 0.07$ [24]. We choose $f_{N}=0.3$ and 0.1 for the purpose of illustration in the following. 


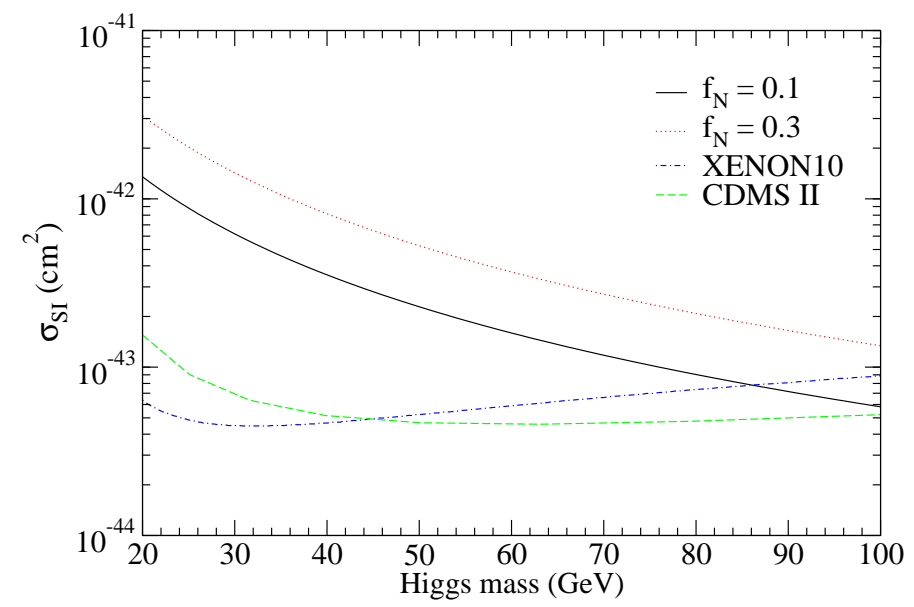

Figure 2: Spin-independent $H N$ scattering cross sections as functions of Higgs mass along with the bounds of CDMS II (dashed, green) and XENON10 (dasd-dotted, blue). The solid (black) and the dotted (red) curves are for two different values of $f_{N}=0.1$ and 0.3 , respectively.

In fig. 2, we show the Higgs-nucleon scattering cross sections as functions of Higgs mass for two different values of $f_{N}=0.3$ and 0.1 with $f_{H}=246 \mathrm{GeV}$. The present experimental upper bounds for the spin-independent WIMP-nucleon cross sections from CDMS II [25] and XENON10 [26] are depicted as well. For $m_{H}=70 \mathrm{GeV}$, the experimental bound is $\sigma_{\mathrm{SI}} \lesssim 5 \times 10^{-44} \mathrm{~cm}^{2}$ at $90 \% \mathrm{CL}$, whereas our prediction is $\sigma_{S I} \simeq(1.2-2.7) \times 10^{-43} \mathrm{~cm}^{2}$ for $f_{N}=(0.1-0.3)$.

This does not necessarily mean that the present model is excluded. The direct detection signals are proportional to the local density of cold dark matter, $\rho_{0}$, which has not been measured by experiments. In most cases including CDMS II and XENON10, the experimental bounds are derived under assuming $\rho_{0}=0.3 \mathrm{GeV} / \mathrm{cm}^{3}$. For a spherical and smooth halo, $\rho_{0}=0.2-0.6 \mathrm{GeV} / \mathrm{cm}^{3}$ seems a reasonable range [3, 4]. Taking the lower value relaxes the constraint. Further it has been argued that $\rho_{0}$ can be as small as $0.04 \mathrm{GeV} / \mathrm{cm}^{3}$ for non-smooth distribution of dark matter in the Galactic halo [27], which makes $m_{H}=70 \mathrm{GeV}$ consistent with the data. On the theoretical side, as mentioned below eq. (77), the $H H \bar{f} f$ coupling constants may be reduced if the mass function $m_{f}\left(\theta_{H}\right)$ has more general $\theta_{H}$-dependence. In this case the Higgs-nucleon cross section is decreased, while the Higgs relic abundance, which depends on both the $H H \bar{b} b$ and $H H W W$ couplings, is kept unchanged by appropriately increasing $m_{H}$. To summarize, it is premature 
to exclude the Higgs dark matter scenario based on the current bounds from CDMS II and XENON10.

Signals from pair annihilation of Higgs bosons in the Galactic halo into two $\gamma$ 's or a $\gamma$ and a $Z$ boson may be seen, too. Expected signals are two (nearly) monochromatic gamma lines of $E_{\gamma}=m_{H}(\simeq 70 \mathrm{GeV})$ and $E_{\gamma}=m_{H}-m_{Z}^{2} /\left(4 m_{H}\right)(\simeq 40 \mathrm{GeV})$. Their rates are estimated to be $\left.\sigma_{\gamma \gamma} v\right|_{v \rightarrow 0} \simeq 4.3 \times 10^{-29} \mathrm{~cm}^{3} / \mathrm{s}$, and $\left.\sigma_{\gamma Z} v\right|_{v \rightarrow 0} \simeq 5.4 \times 10^{-29} \mathrm{~cm}^{3} / \mathrm{s}$ for $m_{H}=70 \mathrm{GeV}$ and $f_{H}=246 \mathrm{GeV}$. Comparing these rates with those in the inert doublet model [19], we expect that such monochromatic gamma rays could be observed by FERMI Gamma-ray Space Telescope (formerly GLAST). However the signals will be less pronounced in our model compared with the inert doublet model, since the annihilation cross section is dominated by $H H \rightarrow b \bar{b}, W^{(*)} W^{(*)}$ in our case, which yield secondary photons with a continuum spectrum.

In this letter we have shown that the Higgs boson becomes absolutely stable in a class of the gauge-Higgs unification models. The stability of the Higgs boson is protected by a new dynamically emerging parity, $H$-parity. Higgs bosons become the cold dark matter in the universe. The observed dark matter density is obtained with $m_{H} \sim 70 \mathrm{GeV}$. Although the direct detection rate for the Higgs-nucleon elastic scattering is found slightly above the current upper bounds, the prediction in the gauge-Higgs unification model may be consistent in view of many uncertainties involved. If the Higgs boson is absolutely stable, the way of finding Higgs bosons in collider experiments must be scrutinized. Higgs bosons appear as missing energies and momenta. We shall come back to these points in future.

\section{Acknowledgments}

We are grateful to P. Gondolo, K. Olive, S. Scopel and Jonghee Yoo for useful discussions and communications. This work was supported in part by Scientific Grants from the Ministry of Education and Science, Grant No. 20244028, Grant No. 20025004, and Grant No. 50324744 (Y.H.), and Grant No. 20244037 (M.T.).

\section{References}

[1] M. Tegmark et al., Phys. Rev. D74 (2006) 123507.

[2] E. Komatsu et al., WMAP Collaboration, Astrophys. J. Suppl. 180 (2009) 330.

[3] G. Jungman, M. Kamionkowski and K. Griest, Phys. Rep. 267 (1996) 195.

[4] G. Bertone, D. Hooper and J. Silk, Phys. Rep. 405 (2005) 279.

[5] A. Djouadi, Phys. Rep. 457 (2008) 1. 
[6] Y. Hosotani, Phys. Lett. B126 (1983) 309.

[7] A.T. Davies and A. McLachlan, Phys. Lett. B200 (1988) 305; Nucl. Phys. B317 (1989) 237.

[8] Y. Hosotani, Ann. Phys. (N.Y.) 190 (1989) 233.

[9] L. Randall and R. Sundrum, Phys. Rev. Lett. 83 (1999) 3370.

[10] Y. Hosotani, K. Oda, T. Ohnuma and Y. Sakamura, Phys. Rev. D78 (2008) 096002, Erratum, ibid. D79 (2009) 079902(E).

[11] Y. Hosotani and Y. Kobayashi, Phys. Lett. B674 (2009) 192. (arXiv:0812.4782[hep-ph])

[12] Y. Sakamura and Y. Hosotani, Phys. Lett. B645 (2007) 442,

Y. Hosotani and Y. Sakamura, Prog. Theoret. Phys. 118 (2007) 935.

[13] Y. Sakamura, Phys. Rev. D76 (2007) 065002.

[14] K. Agashe, R. Contino and A. Pomarol, Nucl. Phys. B719 (2005) 165.

[15] A.D. Medina, N.R. Shah and C.E.M. Wagner, Phys. Rev. D76 (2007) 095010.

[16] N. Haba, M. Harada, Y. Hosotani and Y. Kawamura, Nucl. Phys. B657 (2003) 169; Erratum, ibid. B669 (2003) 381.

[17] Y. Hosotani and M. Mabe, Phys. Lett. B615 (2005) 257.

[18] R. Barbieri, L.J. Hall and V.S. Rychkov, Phys. Rev. D74 (2006) 015007.

[19] M. Gustafsson, E. Lundström, L. Bergström and J. Edsjö, Phys. Rev. Lett. 99 (2007) 041301.

[20] E.W. Kolb and M.S. Turner, The Early Universe, Addison-Wesley, Redwood City, 1990.

[21] G. Belanger, F. Boudjema, A. Pukhov and A. Semenov, Comput. Phys. Commun. 180 (2009) 747.

[22] M.A. Shifman, A.I. Vainshtein and V.I. Zakharov, Phys. Lett. B78 (1978) 443.

[23] J. R. Ellis, K. A. Olive and C. Savage, Phys. Rev. D77 (2008) 065026.

[24] H. Ohki et al., Phys. Rev. D78 (2008) 054502 Phys. Rev. D 78, 054502 (2008).

[25] Z. Ahmed et al. [CDMS Collaboration], Phys. Rev. Lett. 102 (2009) 011301.

[26] J. Angle et al. [XENON Collaboration], Phys. Rev. Lett. 100 (2008) 021303.

[27] M. Kamionkowski and S.M. Koushiappas, Phys. Rev. D77 (2008) 103509. 